verhältnis Vanadin : Sauerstoff beträgt also $1: 3,0$, und die Metavanadinsäure ist somit als 4-basige Tetravanadinsäure, $\mathrm{H}_{4} \mathrm{~V}_{4} \mathrm{O}_{12}$, zu formulieren.

Für die Bildung der oben genannten Dekavanadate werden $1,80 \mathrm{H}_{2} \mathrm{O}$ pro $1 \quad\left(\mathrm{C}_{4} \mathrm{H}_{9}\right)_{3} \mathrm{VO}_{4}$ verbraucht. Die Reaktionsgleichungen lauten also:

$10 \mathrm{R}_{3} \mathrm{VO}_{4}+18 \mathrm{H}_{2} \mathrm{O}+6 \mathrm{~b}=\left(\mathrm{b} \mathrm{H}^{\oplus}\right)_{6} \quad \mathrm{~V}_{10} \mathrm{O}_{28}{ }^{6} \ominus+30 \mathrm{ROH}$,

$10 \mathrm{R}_{3} \mathrm{VO}_{4}+18 \mathrm{H}_{2} \mathrm{O}+5 \mathrm{~b}=\left(\mathrm{b} \mathrm{H}^{\oplus}\right)_{5} \mathrm{HV}_{10} \mathrm{O}_{28}{ }^{5}+30 \mathrm{ROH}$,

$10 \mathrm{R}_{3} \mathrm{VO}_{4}+18 \mathrm{H}_{2} \mathrm{O}+4 \mathrm{~b}=\left(\mathrm{b} \mathrm{H}^{\oplus}\right){ }_{4} \mathrm{H}_{2} \mathrm{~V}_{10} \mathrm{O}_{28}{ }^{4} \odot+30 \mathrm{ROH}$.

Die Dekavanadinsäure weist das Atomverhältnis Vanadin : Sauerstoff $=1: 2,8$ auf und ist als 6-basige Säure, $\mathrm{H}_{6} \mathrm{~V}_{10} \mathrm{O}_{28}$, zu formulieren.

Aus wäßrigen Lösungen kristallisiert mit Pyridin als Anhydrobase das neutrale Pyridiniumsalz dieser Dekavanadinsäure als Dihydrat ${ }^{3}$ aus. Bei der Hydrolyse des Orthovanadinsäure-tert.-butylesters mit feuchtem Äther in Gegenwart von Pyridin (Py.) werden $1,78 \mathrm{H}_{2} \mathrm{O}$ pro $\left(\mathrm{C}_{4} \mathrm{H}_{9}\right)_{3} \mathrm{VO}_{4}$ verbraucht; hier entsteht also das wasserfreie neutrale Dekavanadat $\left(\mathrm{Py} . \mathrm{H}^{\oplus}\right){ }_{6} \mathrm{~V}_{10} \mathrm{O}_{28}{ }^{6 \ominus}$. Erfolgt jedoch die Hydrolyse durch feuchtes Aceton mit Was- sergehalten zwischen 0,5 und $5 \%$, so sind nur $1,70 \mathrm{H}_{2} \mathrm{O}$ pro $1 \quad\left(\mathrm{C}_{4} \mathrm{H}_{9}\right)_{3} \mathrm{VO}_{4}$ erforderlich. Dies bedeutet ein Atomverhältnis Vanadin : Sauerstoff $=1: 2,7$ im aus. fallenden Pyridinium-dekavanadat und läßt darauf schließen, daß das Anion $\mathrm{H}_{2} \mathrm{~V}_{10} \mathrm{O}_{28}{ }^{4} \ominus$ unter besonderen Umständen in der Lage ist, unter Abspaltung einer Wassermolekel in das Anion einer 4-basigen Dekavanadinsäure, $\mathrm{H}_{4} \mathrm{~V}_{10} \mathrm{O}_{27}$, überzugehen; die Hydrolyse wird hier durch die Reaktionsgleichung

$10 \mathrm{R}_{3} \mathrm{VO}_{4}+17 \mathrm{H}_{2} \mathrm{O}+4 \mathrm{Py} .=\left(\mathrm{Py} . \mathrm{H}^{\oplus}\right)_{4} \mathrm{~V}_{10} \mathrm{O}_{27}{ }^{4}+30 \mathrm{ROH}$ beschrieben.

Analoge Untersuchungen über andere Hydrolysevorgänge, die zur Entstehung mehrkerniger, in organischen Lösungsmitteln unlöslicher Verbindungen vom Typus der Polysäuren und Polybasen führen, haben wir bereits in Angriff genommen bzw. möchten wir uns vorbehalten.

3 S. Katzoff u. R. Roseman, J. Amer. chem. Soc. 58, 1785 [1936].

\section{Eine neue Methode zur Wasserbestimmung in organischen Lösungsmitteln}

Von K. F. JAHR und J. Fuchs

Institut für Anorganische Chemie der Freien Universität Berlin

(Z. Naturforschg. 14 b, 471—472 [1959]; eingegangen am 22. Juni 1959)

Die Kenntnis des Reaktionsablaufs der Hydrolyse ${ }^{1}$ von Orthovanadinsäure-tert.-butylester, $\left(\mathrm{C}_{4} \mathrm{H}_{9}\right)_{3} \mathrm{VO}_{4}$, der zusammen mit einer Anhydrobase in wasserfeuchte organische Lösungsmittel eingebracht wird, zu unlöslichen Polyvanadaten führte zur Ausarbeitung eines neuen, recht exakten Verfahrens der Aquametrie.

Die Bestimmung erfolgt in einer einfachen, geschlossenen Glasapparatur, die den Zutritt von Luftfeuchtigkeit während der analytischen Operation ausschließt, durch Einwirken der im Überschuß zugegebenen Reagenzlösung auf ein genau abgemessenes Volumen des zu untersuchenden Lösungsmittels. Als Reagenzlösung dient in der Regel ein Gemisch gleicher Volumina einer Lösung des Esters in Testbenzin und ammoniakgesättigten Chloroforms. Der entstandene Polyvanadat-Niederschlag wird filtriert und mit wasserfreiem Äther gewaschen. Er wird sodann, außerhalb der Bestimmungsapparatur, in verdünnter, wäßriger Schwefelsäure gelöst. In der Lösung wird durch Titration ${ }^{2}$ mit Eisen(2) sulfat die Menge des Vanadins bestimmt. Aus dieser wird der Wassergehalt des untersuchten Lösungsmittels mit Hilfe der Reaktionsgleichung

$$
4 \mathrm{R}_{3} \mathrm{VO}_{4}+4 \mathrm{NH}_{3}+8 \mathrm{H}_{2} \mathrm{O}=\left(\mathrm{NH}_{4}\right)_{4} \mathrm{~V}_{4} \mathrm{O}_{12}+12 \mathrm{ROH}
$$

$\left(\mathrm{R}=\mathrm{C}_{4} \mathrm{H}_{9}\right)$ stöchiometrisch berechnet $\left(\mathrm{H}_{2} \mathrm{O}: \mathrm{V}=2,0: 1\right)$.

Für die Wasserbestimmung in Aceton muß an Stelle des Ammoniaks eine tertiäre organische Anhydrobase

1 K. F. JAHR u. J. Fuchs, Z. Naturforschg. 14 b, 468 [1959]. treten, weil Aceton in Gegenwart von Ammoniak zu Kondensations-Reaktionen neigt. Als geeignet erwies sich Acridin (Acr.). Die Hydrolyse führt gemäß den Reaktionsgleichungen:

$$
\begin{aligned}
& 10 \mathrm{R}_{3} \mathrm{VO}_{4}+4 \text { Acr. }+18 \mathrm{H}_{2} \mathrm{O}=\left(\text { Acr. } \mathrm{H}_{4} \mathrm{H}_{2} \mathrm{~V}_{10} \mathrm{O}_{28}+30 \mathrm{ROH},\right. \\
& 10 \mathrm{R}_{3} \mathrm{VO}_{4}+5 \text { Acr. }+18 \mathrm{H}_{2} \mathrm{O}=(\text { Acr. } \mathrm{H})_{5} \mathrm{HV}_{10} \mathrm{O}_{28}+30 \mathrm{ROH}
\end{aligned}
$$

zu zwei sauren Dekavanadaten; Rk. (2) spielt sich vorzugsweise in wasserärmerem Aceton $(<2,5$ Vol. $\%$ $\left.\mathrm{H}_{2} \mathrm{O}\right)$, Rk. (3) in wasserreicherem Aceton $(>2,5$ Vol. $\%$ $\mathrm{H}_{2} \mathrm{O}$ ) ab. Das stöchiometrische Verhältnis von $\mathrm{H}_{2} \mathrm{O}: \mathrm{V}$ wird dadurch nicht beeinflußt, es beträgt in jedem Fall $1,8: 1$.

Der Zeitbedarf für eine Bestimmung beträgt 15 bis 20 Minuten.

Die neue Methode eignet sich besonders zur Bestimmung sehr kleiner Wassergehalte z. B. in Kohlenwasserstoffen, Alkylhalogeniden, Äthern, Ketonen, Estern und solchen Alkoholen, in denen die Polyvanadate praktisch unlöslich sind. Sie ist daher nicht anwendbar in Methanol und Glycerin. Bei Äthanol macht sich der Einfluß der Löslichkeit erst bei dem Versuch, Wasserspuren zu bestimmen, störend bemerkbar; zur Kontrolle des Trokkengrades von abs. Äthanol ist das Verfahren also nicht geeignet.

Besondere Vorteile des beschriebenen Verfahrens sind der stöchiometrische Ablauf der Hydrolyse-Reaktionen, durch den Eichbestimmungen unnötig werden, die Haltbarkeit und der vergleichsweise geringe Preis der Reagenzlösung sowie der Umstand, daß die Kenntnis ihres Titers zur Durchführung der Bestimmung nicht erforderlich ist.

Tab. 1 läßt den Grad der Reproduzierbarkeit von Wasserbestimmungen erkennen, die nach dieser Methode an verschiedenen Lösungsmitteln durchgeführt worden sind. Mit welcher Genauigkeit Wassermengen bestimmt wurden, die in Lösungsmittel bekannten Was- 
sergehalts zusätzlich eingewogen waren, zeigt Tab. 2 . Sie läßt zugleich die Proportionalität der Analysenwerte erkennen.

\begin{tabular}{|l|c|c|c|}
\hline $\begin{array}{c}\text { untersuchtes } \\
\text { Lösungsmittel }\end{array}$ & $\begin{array}{c}\text { vorgelegtes } \\
\text { Volumen [ml] }\end{array}$ & $\begin{array}{c}\text { Wassergehalt } \\
{[\%]}\end{array}$ & $\begin{array}{c}\text { Reproduzier- } \\
\text { barkeit [\%] }\end{array}$ \\
\hline Testbenzin & 10 & 0,0083 & $\pm 0,0001$ \\
Benzol & 10 & 0,0252 & $\pm 0,0004$ \\
Chloroform & 10 & 0,119 & $\pm 0,001$ \\
Äther & 2 & 0,433 & $\pm 0,003$ \\
Aceton 1 & 1 & 5,68 & $\pm 0,02$ \\
2 & 3 & 1,278 & $\pm 0,004$ \\
3 & 10 & 0,270 & $\pm 0,001$ \\
Äthanol 1 & 1 & 5,09 & $\pm 0,01$ \\
2 & 2 & 1,299 & $\pm 0,003$ \\
3 & 4,5 & 0,1840 & $\pm 0,0003$ \\
\hline
\end{tabular}

Tab. 1. Reproduzierbarkeit der Wasserbestimmung.

2 J. Knop u. O. Kubelková-Knopová, Z. analyt. Chem. 122, 183 [1941].

\section{Entcarbobenzoxylierung von Aminosäuren und Derivaten mit wasserfreier Trifluoressigsäure}

Von Friedrich Weygand und Wolfgang Steglich

Organisch-Chemisches Institut der Technischen Hochschule München, München 2

(Z. Naturforschg. 14 b, 472—473 [1959] ; eingegangen am 1. Juni 1959)

Bei der Umsetzung von Glycin mit Trifluoressigsäureanhydrid in Gegenwart von Glycinbenzylester wurde die Bildung von Trifluoressigsäurebenzylester beobachtet ${ }^{1}$.

Wir haben nun die Einwirkung von wasserfreier Trifluoressigsäure auf Benzyloxycarbonyl-Verbindungen, die sogenannten Carbobenzoxy-Verbindungen, von Aminosäuren, Peptiden und deren Estern untersucht. Es zeigte sich, daß beim Erhitzen unter Rückfluß schnell Kohlendioxyd-Entwicklung stattfindet. Neben Trifluoressigsäurebenzylester, der unter den Reaktionsbedingungen teilweise mit sich selbst zu nicht näher untersuchten, fluoreszierenden Produkten reagiert, entstehen die trifluoressigsauren Salze von Aminosäuren, Peptiden und deren Estern. Aus diesen sind die freien Aminosäuren und Peptide sowie die Hydrochloride der Aminosäure- und Peptid-ester leicht zu isolieren. Auch die Hydrochloride von aktivierten Estern kann man auf diese Weise erhalten. Die Ausbeuten liegen meist zwischen 80 und $90 \%$ der Theorie.

Der als Nebenprodukt entstehende Trifluoressigsäurebenzylester wirkt in wasserfreier Trifluoressigsäure benzylierend und kann mit aromatischen Aminosäuren sowie mit Benzylestern und Benzylschutzgruppen reagieren. Er verhält sich dabei ähnlich wie der Toluolsulfonsäurebenzylester ${ }^{2}$. Durch Zusatz einer elektrophil leicht substituierbaren aromatischen Verbindung, wie Phenol, Resorcin oder Anisol, lassen sich diese Nebenreaktionen

1 F. Weygand u. E. Leising, Chem. Ber. 87, 249 [1954].

2 Z. FöLdi, Ber. dtsch. chem. Ges. 61, 1609 [1928].

\begin{tabular}{|l|c|c|c|}
\hline $\begin{array}{c}\text { untersuchtes } \\
\text { Lösungsmittel }\end{array}$ & $\begin{array}{c}\text { Einwaage } \\
\text { pro 100 ml } \\
{[\mathrm{g}]}\end{array}$ & $\begin{array}{c}\text { gefunden } \\
\text { in l ml } \\
{[\mathrm{mg}]}\end{array}$ & $\begin{array}{c}\text { Fehler } \\
{[\mathrm{mg}]}\end{array}$ \\
\hline Äthanol & 1,2944 & 12,99 & $+0,05$ \\
& 2,1354 & 21,48 & $+0,13$ \\
& 5,0894 & 50,90 & $+0,01$ \\
Aceton & 1,0412 & 10,48 & $+0,07$ \\
& 2,0980 & 21,04 & $+0,06$ \\
& 2,7892 & 27,74 & $-0,15$ \\
& 3,2130 & 32,16 & $+0,03$ \\
Pyridin & 5,4690 & 54,48 & $-0,21$ \\
& 1,0989 & 11,08 & $+0,09$ \\
Essigester & 2,0276 & 20,25 & $-0,03$ \\
& 3,1340 & 31,50 & $+0,16$ \\
Dioxan & 0,1144 & 1,16 & $+0,02$ \\
\hline
\end{tabular}

Tab. 2. Absolutgenauigkeit und Proportionalität der Wasserbestimmung.

\begin{tabular}{|c|c|c|c|c|}
\hline $\begin{array}{l}\text { Carbobenzoxyverbindung } \\
(\mathrm{Z}=\text { Carbobenzoxy-Rest) }\end{array}$ & 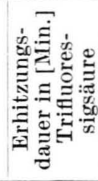 & 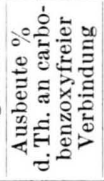 & $\begin{array}{l}\text { Zusatz } \\
\text { von }\end{array}$ & 营 \\
\hline $\begin{array}{l}\text { Z-Glycin } \\
\text { Z-DL-Phenylalanin } \\
\text { Z-DL-Phenylalanin } \\
\text { Z-O-Benzyl-DL-serin } \\
\text { Z-S-Benzyl-L-cystein } \\
\text { Z-S-Benzyl-L-cystein } \\
\text { Z-L-Asparagin } \\
N \text {-Z-L-Tyrosin } \\
N \text {-O-Bis-Z-L-tyrosin } \\
\text { Z-O-Benzyl-L-tyrosin } \\
\text { Bis-Z-L-histidin } \\
\text { Z-Glycyl-DL-leucin } \\
\text { Z-Glycyl-glycin } \\
\text { Z-Glycyl-DL- } \\
\text { phenylalanin } \\
\text { Z-Glycyl-DL-alanyl- } \\
\text { glycin } \\
\text { Z-L-Alanyl-L-valin } \\
\text { Z-L-Leucyl-glycin- } \\
\text { äthylester } \\
\text { Z-Glycin-thiophenyl- } \\
\text { ester } \\
\text { Z-L-Asparaginsäure- } \\
\alpha-p \text {-nitro-phenyl- } \\
\text { ester- } \beta \text {-methylester } \\
\text { Z-Glycyl-DL-phenyl- } \\
\text { alanin-benzylester } \\
\text { Z-Glycyl-DL-phenyl- } \\
\text { alanin-benzylester } \\
\text { Z-Glycyl-DL-phenyl- } \\
\text { alanin-benzylester }\end{array}$ & $\begin{array}{r}90 \\
30 \\
60 \\
23 \\
60 \\
60 \\
20 \\
30 \\
30 \\
30 \\
150 \\
60 \\
12\end{array}$ & $\begin{array}{l}88 \\
85 \\
92 \\
77 \\
63 \\
92 \\
94 \\
98 \\
92 \\
41^{1} \\
51 \\
78 \\
77 \\
83 \\
84 \\
56 \\
88^{2} \\
88^{2} \\
93^{3}\end{array}$ & $\begin{array}{l}\text { Resorcin } \\
\text { Anisol } \\
-* \\
- \\
\text { Anisol } \\
- \\
\text { Resorcin } \\
\text { Anisol } \\
\text { Anisol } \\
\text { Resorcin } \\
\quad- \\
\quad- \\
\text { Resorcin } \\
\quad \\
- \\
- \\
- \\
-\end{array}$ & $\begin{array}{l}\mathrm{B} \\
\mathrm{B} \\
\mathrm{B} \\
\mathrm{B} \\
\mathrm{B} \\
\mathrm{B} \\
\mathrm{B} \\
\mathrm{B} \\
\mathrm{B} \\
\mathrm{B} \\
\mathrm{B} \\
\mathrm{B} \\
\mathrm{B}\end{array}$ \\
\hline
\end{tabular}

Tab. 1. Entcarbobenzoxylierung von Aminosäuren und Derivaten. ${ }^{*}$ Anisolzusatz ist empfehlenswert. ${ }^{1}$ Neben $51 \%$ Tyrosin. ${ }^{2}$ Als Hydrochlorid isoliert. ${ }^{3}$ Als Trifluoracetat isoliert. ${ }^{4}$ Neben $45 \%$ Glycyl-DL-phenylalanin. ${ }^{5}$ Glycyl-pL-phenylalanin. 